\title{
Armamento aéreo: una mirada hacia el futuro
}

| Fecha de recibido: 28 de junio del 2021 | Fecha de aprobación: 23 de septiembre del 2021 |

Leonardo de Jesús
Mesa Palacio
Magíster en Sistemas
Automáticos de Producción
Docente, Servicio Nacional de Aprendizaje (SENA)
Colombia
Rol del investigador: escritura
https://orcid.org/0000-0002-4903-723X
$\square$ lomesap@sena.edu.co

Abdon Estibenson Uribe Taborda

Magíster en Seguridad Operacional Escuela de Postgrados Fuerza Aérea Colombiana Colombia

Rol del investigador: escritura https://orcid.org/0000-0003-4058-4365 $\triangle$ abdon.uribe@fac.mil.co 


\section{Armamento aéreo: una mirada hacia el futuro}

\author{
Air Weapons: \\ A Look into the Future
}

Resumen: Este artículo tiene por objetivo realizar un análisis descriptivo del armamento destinado a la batalla aérea a lo largo de los siglos xx y xXI, para entender el impacto de estas armas, la superioridad aérea militar de los países y su necesidad de avance tecnológico en el ámbito del armamento en los escenarios de conflicto. En su historia, el ser humano ha tenido que enfrentar permanentemente la resolución de conflictos que se han creado por diferencias a nivel social, político, económico y militar. Cuando estos problemas exceden el marco de las discusiones, se requieren tomar medidas más fuertes para restablecer el orden social. Así, los avances tecnológicos en el ámbito del armamento aéreo han desempeñado un papel protagónico y han permitido a los Estados mantener el orden en los casos de extrema dificultad, en pro de los intereses de una comunidad.

Palabras clave: armamento; batalla aérea; historia; operativo; adversario; tecnología; futuro.

Abstract: This article presents a descriptive analysis of the weaponry used for air combat throughout the 20th and 21st centuries in order to understand the impact of these devices, the military air supremacy of some countries, and the need for technological advancement in certain conflict settings. Historically, human beings have permanently faced the resolution of conflicts created by differences at social, political, economic and military levels. When these events exceed the scope of diplomatic discussions, stronger measures are required to reestablish social order. Hence, technological advances in the field of air weapons have played a leading role, allowing States to maintain order in cases of extreme difficulty in the bests interests of a society.

Keywords: weapons; air combat; history; operation; adversary; technology; future.

Resumo: Este artigo tem como objetivo realizar uma análise descritiva do armamento destinado ao combate aéreo ao longo dos séculos XX e XXI, para compreender o impacto dessas armas, a superioridade aérea militar dos países e sua necessidade de avanço tecnológico, armas em ambientes de conflito. Em sua história, o ser humano teve que enfrentar permanentemente a resolução de conflitos que se originaram das diferenças de nível social, político, econômico e militar. Quando esses problemas vão além do escopo das discussões, medidas mais fortes são necessárias para restaurar a ordem social. Assim, os avanços tecnológicos no campo das armas aéreas têm desempenhado um papel de liderança e têm permitido aos Estados manter a ordem em casos de extrema dificuldade, pelo interesse de uma comunidade.

Palavras-chave: armas; batalha aérea; história; operacional; adversário; tecnologia; futuro. 
Las principales funciones de los militares, líderes y gerentes se fundamentan en la toma de decisiones y en la búsqueda permanente del cumplimiento de los objetivos y metas trazadas por un país, en donde prevalecen los intereses colectivos antes que los personales; esto visto como una manera de afrontar los nuevos retos para mantener la seguridad. El término seguridad es aplicado según el contexto al cual está dirigido, lo que da paso a niveles de aplicabilidad de este concepto, en los cuales los Estados tratan de cuidar su integridad territorial para conservar sus intereses particulares y, por ende, el bien común de su población mediante la utilización de todos los medios para lograrlo, entre ellos las capacidades bélicas que son fundamentales para garantizar la percepción de seguridad de una población.

Colombia, y más específicamente la Fuerza Aérea Colombiana, tendrá que adaptar el poder aéreo y las doctrinas de acción integral a nuevos retos en materia de seguridad, con una serie de desafíos que deben ser advertidos desde la actualidad, sin esperar a que surjan nuevas amenazas (Strong \& Jaramillo, 2014).

\section{Problema de investigación}

La seguridad es lo principal en las agendas sobre relaciones internacionales que tratan de abordar las naciones que buscan proporcionar, como instrumento político, la sensación de seguridad a las poblaciones. Esta situación cobra fuerza cuando se muestran estadísticas de hechos relevantes en contra de esas migraciones que buscan ser mediáticas y protagonistas ante hechos políticos, sociales y culturales que requieren de las aplicaciones de la seguridad y de la identificación de los fenómenos de globalización para determinar las nuevas amenazas que pueden ser combatidas con la adquisición y modernización del armamento con que cuenta un país para defender sus intereses y los de su comunidad.

La historia de inicios del siglo $\mathrm{xx}$ ha demostrado que el éxito en las guerras se fundamenta en los avances y desarrollos en la capacidad militar; ejemplo de ello es la Primera Guerra Mundial, en la cual se utilizó la radio para las comunicaciones, mediante la radiogoniometría, y la radio interceptación en la determinación de posiciones y movimientos de las tropas de los países protagonistas. Otro ejemplo se puede encontrar en la Segunda Guerra Mundial, evento el que se dieron diversos descubrimientos electrónicos e iniciaron las batallas en el espectro electromagnético. A esto se le denominó la Guerra Electrónica (EW). Además, la batalla en el espacio aéreo demandó de la utilización de equipamiento de gran importancia dentro de las aeronaves para afrontar los retos que demandaban las nuevas necesidades de transformación en la utilización constante del armamento con fines defensivos (Michavila, 1984).

Para el caso de Colombia, es necesario continuar con la utilización y modernización de su capacidad bélica aérea para consolidar los fines del Estado y la finalización de la guerra, que, tras la desmovilización de las FARC, se prevé que surjan nuevas necesidades de transformación por parte de la fuerza pública de acuerdo con los cambios en el panorama nacional. Esto obliga al Estado a redefinir sus funciones y dispositivos, entre los que se encuentran disminuciones del presupuesto requerido para la defensa y seguridad nacional (Leal, 2017).

\section{Metodología}

Este artículo presenta los temas mediante una investigación de tipo descriptiva con un enfoque cualitativo, que permitirá utilizar la recolección de datos históricos por medio de una revisión de la literatura sobre la utilización del armamento aéreo en diversas aeronaves y escenarios, desde su invención hasta su aplicación, así como las capacidades y características del armamento utilizado según su necesidad bélica, que permita "adquirir y cuantificar información sobre las variables a las que se refieren" (Hernández Sampieri et al., 2010).

Para llevar a cabo esta investigación, se tendrán en cuenta un marco histórico que ilustre al lector, mediante imágenes, texto y conceptos de actualización 
de capacidades militares y superioridad, sobre los avances tecnológicos logrados a través de necesidades de mejoras en el armamento utilizado en las aeronaves de ataque. Esto busca generar planteamientos que serán soportados en el estudio cualitativo de las fuentes consultadas de manera cronológica sobre el proceder de la utilización de las armas bélicas en las plataformas aéreas (Hernández Sampieri et al., 2010).

En la primera fase de la investigación, se relacionarán las primeras experiencias de la utilización del arsenal bélico en las aeronaves y la importancia que estas tuvieron en los periodos en que se generaron, por medio de una descripción de su implementación (Fernández, 2015).

En la segunda fase, se abordarán conceptos de seguridad y defensa para indicar al lector los temas que deben ser tenidos en cuenta al analizar la importancia de que prevalezcan capacidades disuasivas para afrontar situaciones que se presenten en contextos no alineados a las realidades deseadas, como en el caso de los grupos ilegales, brotes de subversión o tensiones diplomáticas (Cobo, 2014). Posteriormente, se realizará un análisis que busca llamar la atención sobre la necesidad de mantener en constante actualización y desarrollo la implementación de nuevas tecnologías en el área del armamento para las aeronaves utilizadas en la defensa de la seguridad nacional de un país (Pinzón, 2020).

\section{Reseña histórica}

Los hermanos Wright realizaron el primer vuelo victorioso en su avión llamado Flyer. Este gran invento abrió la posibilidad de disminuir las distancias entre los hombres, los continentes y los países para realizar intercambios comerciales y de aprendizaje. En los primeros años de la aviación, las aeronaves fueron diseñadas con el único propósito de volar; sin embargo, la mayoría de las aeronaves fueron empleadas para fines de exhibición, carreras o eventos tipo espectáculo. Los ejércitos no fueron ajenos a esta situación, y con el paso del tiempo empezaron a utilizar la aviación con el propósito de observación, transporte y fines bélicos. En 1909, el mayor italiano Guilio Douhet expresó que el uso de aeronaves sería un factor importante en el éxito de futuras guerras. Años después, en 1911, el ejército italiano desplegó operacionalmente esta idea durante la guerra entre Italia y Libia para el reconocer y posteriormente bombardear (Ananthasayanam et al., 2005).

El uso táctico y estratégico de las aeronaves se expandió con el inicio de la Primera Guerra Mundial; las demandas de la aviación de combate, junto con la constante competencia por la superioridad aérea, pasaron del desarrollo de aeronaves de observación y diversión -1903- a vehículos altamente útiles y versátiles, diseñados para cumplir papeles específicos de combate al final de la guerra - por ejemplo, en noviembre de 1918- (Loftin, 1987).

En las primeras batallas aéreas, los pilotos se quitaban de la espalda el fusil y abrían fuego, sin dar en el blanco, y se retiraban cuando se acababa la munición. Por lo tanto, se buscó la manera de mejorar la estrategia instalando una ametralladora fija en la aeronave; algunas versiones del Bristol Scout colocaban la ametralladora direccionada lateralmente.

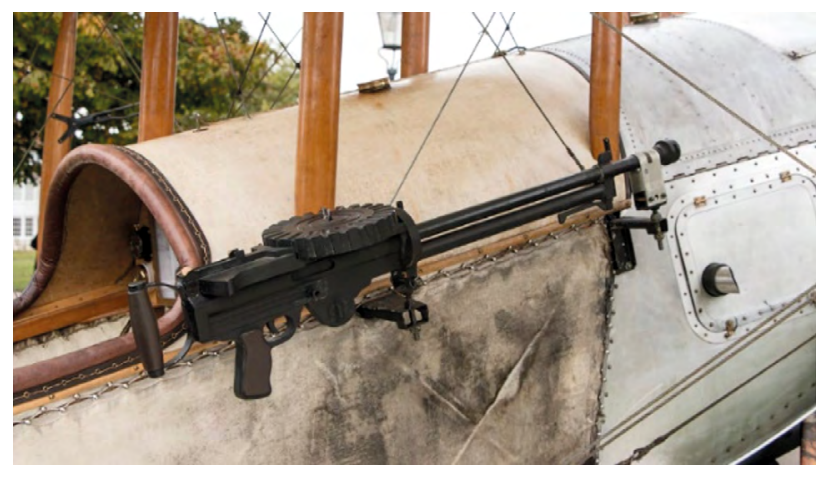

Figura 1. Aeronave Bristol Scout con ametralladora direccionada lateralmente

Fuente: Hiveminer (2020).

Otros aviones, como las versiones iniciales del Neieuport 17 (figura 2), montaban la ametralladora en su plano superior, y los aviones de hélice impulsora, como el Hadvilland DH2 (figura 3), tenían la hélice en la parte posterior, con lo cual el piloto podía manipular el arma. Los tres métodos demostraron ser muy ineficientes. 


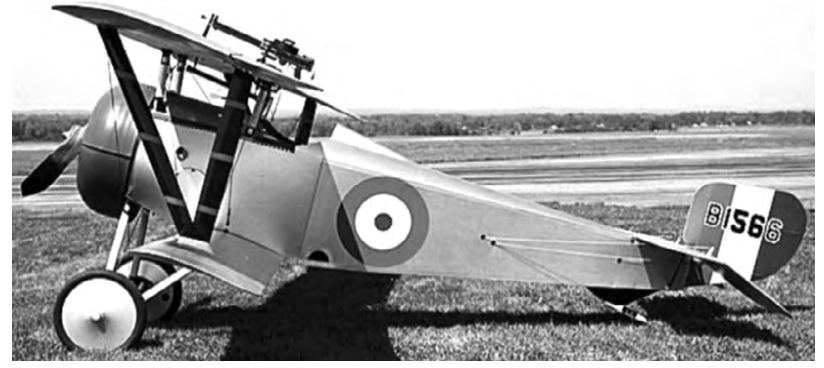

Figura 2. Aeronave Neieuport 17 con ametralladora en el plano superior Fuente: SilverHawkAuthor (2020).

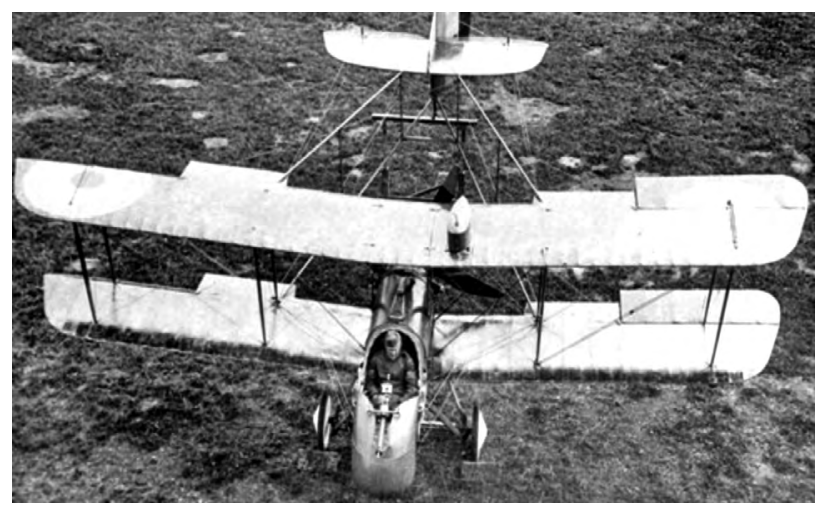

Figura 3. Aeronave Hadvilland DH2 con ametralladora Lewis.303 sobre nariz Fuente: Bae Systems (2020).

La primera solución se dio cuando el francés Roland Garros, deportista y aviador, hizo instalar en su avión una ametralladora sobre el capó; posteriormente, el ingeniero holandés Anthony Fokker perfeccionó el sistema, y la ametralladora únicamente se disparaba cuando la hélice pasaba por determinado punto (Zimmerman, 2019) (figura 4).

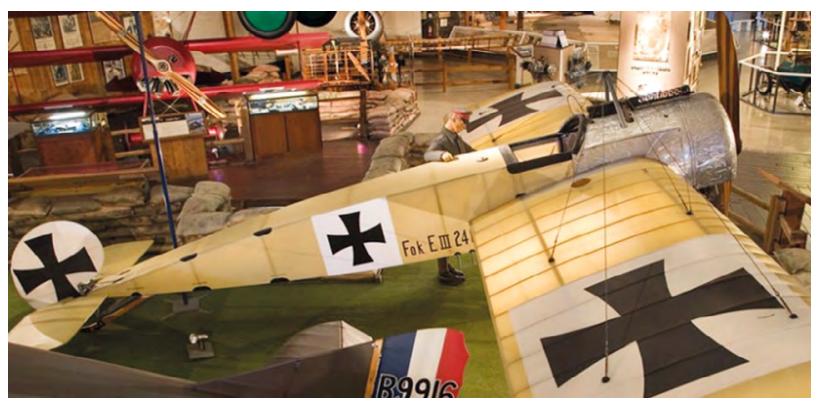

Figura 4. Aeronave Fokker E-III Eindecker con ametralladora de hélice Fuente: San Diego Air \& Space Museum (2020).
Otras armas usadas en la Gran Guerra fueron los dardos aéreos de acero, inventados por los franceses (figura 5). Tanto en el caso de los dardos como en el de las bombas, el piloto podía llevar las cargas en la cabina y luego soltarlas sobre el objeto. En 1918, los bombarderos se habían convertido en aviones pesados con múltiples motores que podían arrojar más de una tonelada de bombas en la retaguardia del adversario; al finalizar la guerra, las naciones eran conscientes del poder del armamento aéreo, y su presencia sería necesaria para futuros escenarios de conflicto bélico.

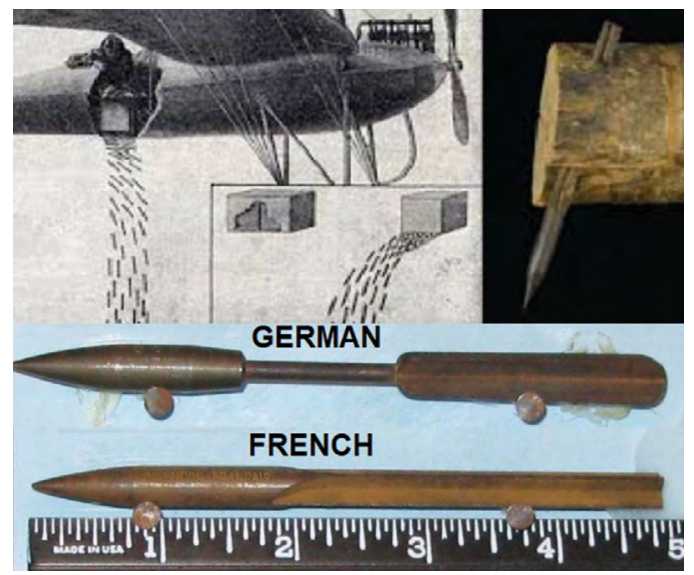

Figura 5. Dardos aéreos de la Gran Guerra Fuente: WonderfulEngineering (2020).

Tras la guerra, hubo una gran depresión económica en el mundo. Por tal motivo, el armamento sobrante fue reciclado y se le realizaron algunos cambios para acondicionarlo a las nuevas necesidades; tal es el caso de los aviones británicos que, como montura de las ametralladoras gemelas Lewis, empleaban el anillo Scarff - patentado por William Scarff Frederick en 1920-, dispositivo que facilitaba los movimientos del apuntador (figura 6). Este dispositivo surgió como una innovación en el desarrollo de la aeronave Sidestrand, lo cual condujo a más pedidos de producción, gracias las dificultades que experimentaron los artilleros del Escuadrón 101 al apuntar sus cañones Lewis montados en anillos Scarff mientras luchaban contra la estela de un avión de tan alto rendimiento. Las altas velocidades alcanzadas por los cazas modernos y el Sidestrand (figura 7) -240 a 300 kilómetros por 
hora- dificultaron que los artilleros apuntasen sus pistolas Lewis montadas en anillos Scarff expuestas como estaban, y que pudieran cambiar los tambores de los cargadores con los dedos congelados. Esta situación a menudo los hacía caer, ocasionando que las hélices se dañaran. Los días de los bombarderos con cabinas abiertas y posiciones de cañones estaban llegando a su fin (Brew, 2020).

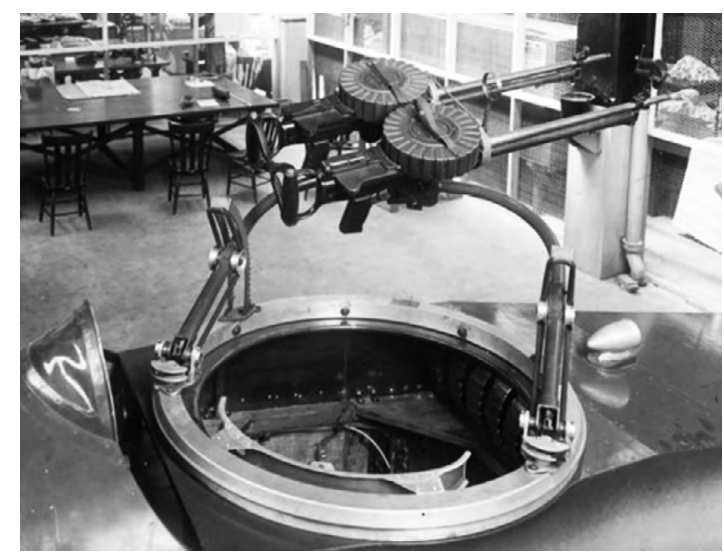

Figura 6. Anillo SCARFF

Fuente: Wright State University (2020).

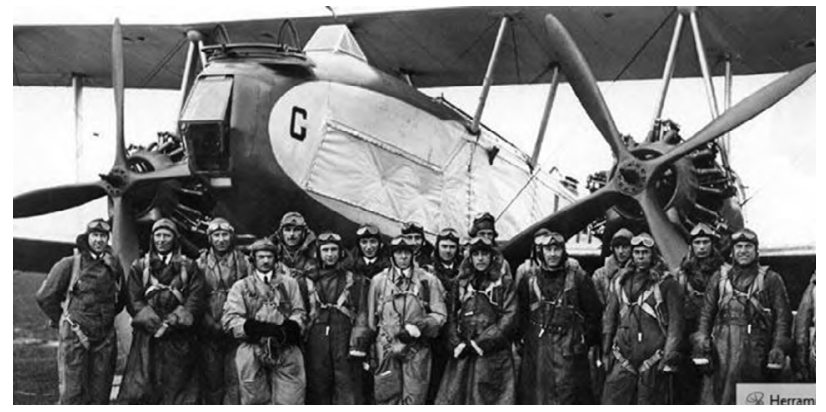

Figura 7. Equipo Sidestrand

Fuente: Militarylmages (2007).

En 1932, se realizaron mejoras a la aeronave Sidestrand, con el fin de proteger al artillero sin comprometer las prestaciones del avión. Las mejoras aparecieron en la aeronave Overstrand. Para superar los problemas del artillero de nariz, el Overstrand se equipó con una torreta de morro cerrada y motorizada que montaba un solo cañón Lewis. El Overstrand fue el primer avión de la Royal Air Force (RAF) en tener una torreta motorizada (figura 8).

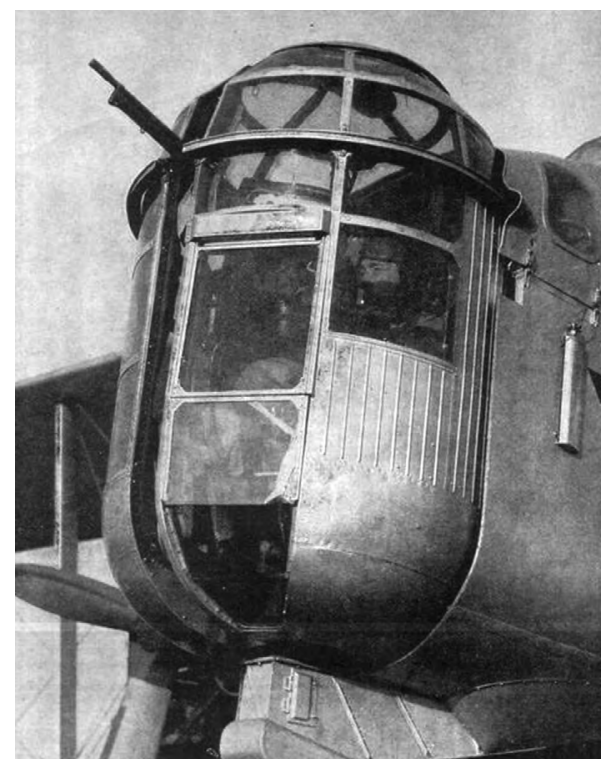

Figura 8. Torreta de cañón Paul Boulton Overstrand Fuente: Richard (2009).

Más adelante, aparecieron las primeras torretas neumáticas, como la utilizada en el bombardero B-10, equipado con el cañón Browning de 0,303 -torreta tipo 130-, el cual posteriormente fue instalado en el avión Boulton Paul Defiant P.82 - caza diurno y nocturno- de la RAF. El B-10 fue el primer bombardero monoplano "moderno" completamente metálico producido en cantidad; presentaba elementos innovadores, como un tren de aterrizaje retráctil, una torreta giratoria y cabinas cerradas. El diseño avanzado de Martin hizo que el B-10 fuera $50 \%$ más rápido que los bombarderos biplanos contemporáneos, y tan rápido como la mayoría de los cazas (Unidos, 2015).

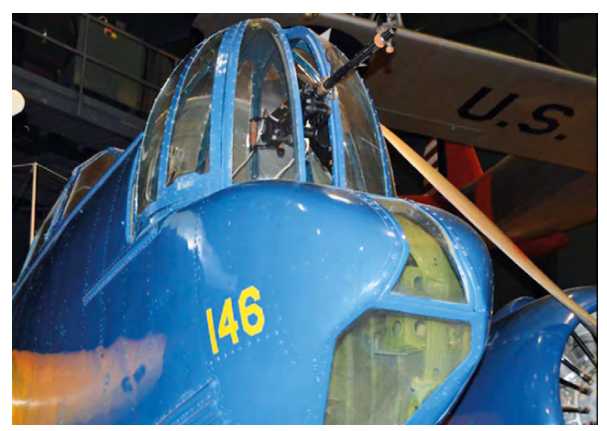

Figura 9. Martin B-10 en la galería "Early Years" del Museo Nacional de la Fuerza Aérea de los Estados Unidos Fuente: Unidos (2015). 
El momento cero para las aeronaves tipo caza se dio en 1937, año en el que la empresa Boulton Paul recibió la orden de fabricación del P.82 Dtfial, que meses más tarde se convertiría en el P.82 Defiant, con su icónica torreta -Torreta Boulton-Paul Type A- (figura 10).
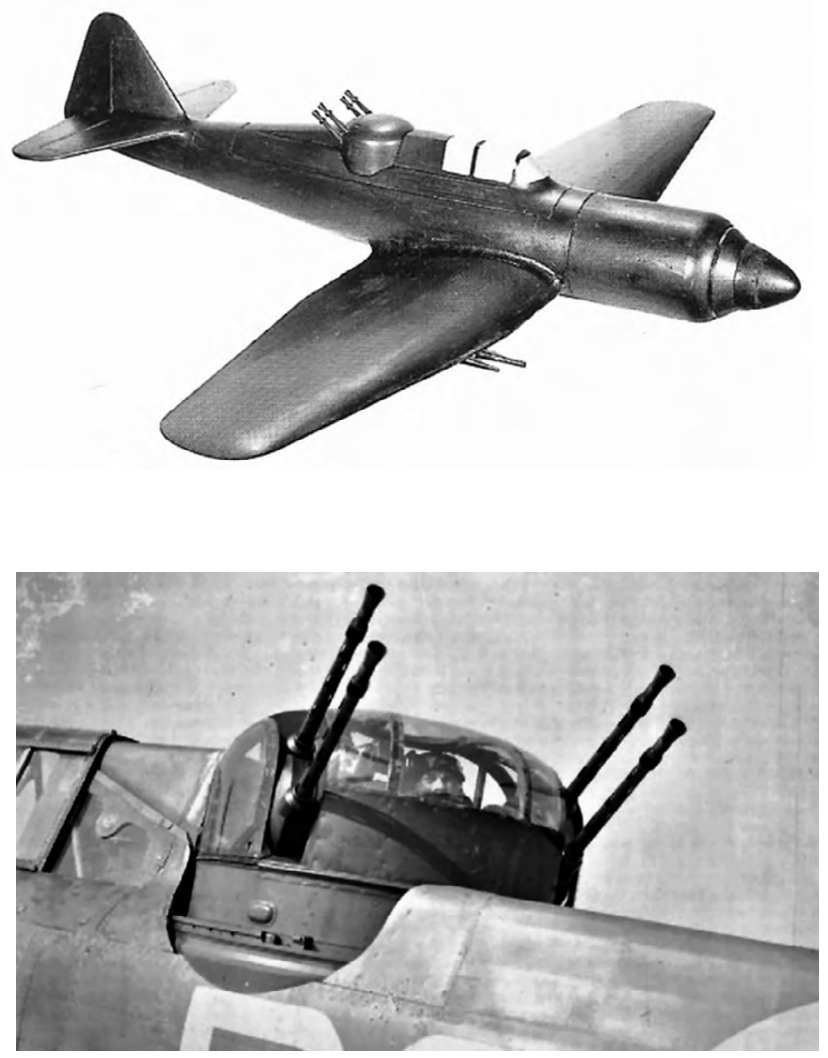

Figura 10. Torreta Boulton-Paul Type A Fuente: Brew (1993).

La aviación militar llegó a su apogeo en la Segunda Guerra Mundial, lo que se tradujo en una batalla de mayor poder aéreo. Uno de los elementos que contribuyó a la derrota de los nazis fue el uso del Boeing B-17, un bombardero pesado y fiable; sus últimas versiones tenían una capacidad de carga de $8.000 \mathrm{~kg}$ de bombas y hasta 13 ametralladoras - ametralladoras Browning M2 de 12,7 mm con 6.380 cartuchos-. El reportero del Seattle Times, Richard Smith, apodó al nuevo avión, con sus numerosos soportes de ametralladora, "Flying Fortress", un nombre que Boeing adoptó y registró rápidamente (Boeing, 2020).

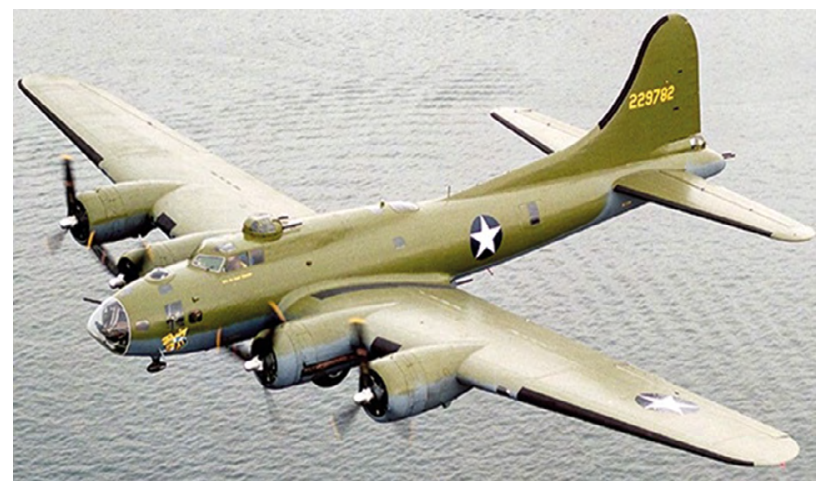

Figura 11. Boeing B-17 Flying Fortress

Fuente: The Museum of Fligth (2020).

Finalizando la Segunda Guerra Mundial, y como respuesta a la aparición de los bombarderos armados, en 1954 se desarrolló un cañón automático conocido como ADEN - ADE del Armament Development Establishment, con $\mathrm{N}$ para Enfield, ciudad donde se estableció su producción-. Este cañón de 30 mm fue instalado en más de una decena de aeronaves, iniciando por el Hawker Hunter, English Electric Lightning, Gloster Javelin, entre otros.

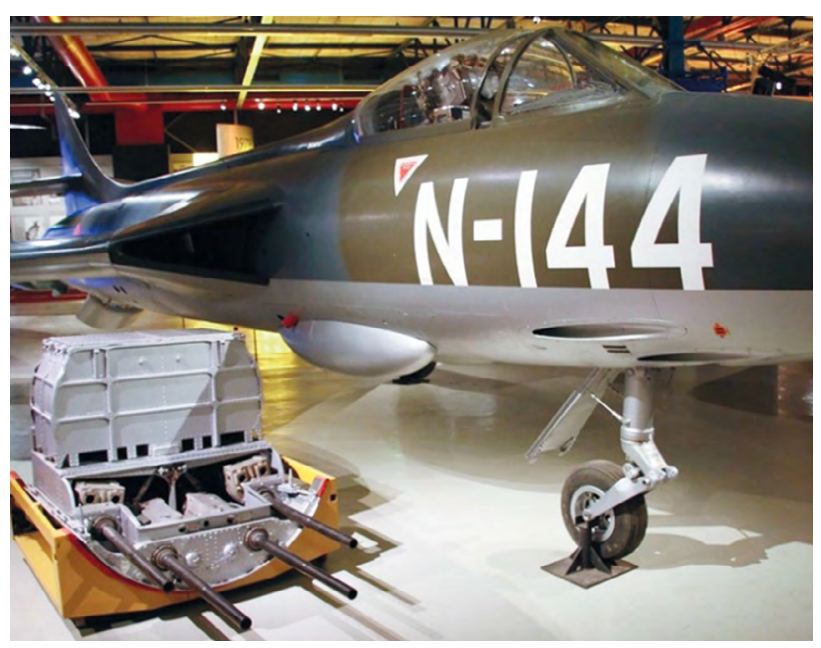

Figura 12. Paquete removible de cañones de $30 \mathrm{~mm}$ ADEN, instalado en Hawker Hunter F.4 (N-144)

Fuente: Wageningen (2008).

Sin embargo, ningún armamento de los mencionados se compara con las bombas atómicas, lanzadas por primera y última vez, en agosto de 1945 sobre 
Hiroshima y Nagasaki ${ }^{1}$, acabando con la vida de unos 200.000 seres humanos a causa de la explosión y la exposición a la radiación. Estados Unidos acreditó el uso de las bombas razonando que, por inhumano que fuera, se lograba que la batalla terminara rápidamente (Fernández, 2015).

Finalizando la Segunda Guerra Mundial, Estados Unidos y la URSS llegaron a ciertos acuerdos, pero en pocos años se vieron envueltos en conflictos de origen político, social y militar. En la década de los sesenta se inició la competencia de armamentos nucleares, durante la cual la Unión Soviética colocó en órbita un satélite artificial llamado Sputnik y lanzó su primer misil balístico intercontinental, lo que tuvo una gran significación tecnológica (Fernández, 2015).

En aquel momento se generó un ambiente de tensión y los norteamericanos empezaron a seguir el programa Single Integrated Operation Plan (SIOP) ${ }^{2}$, que indicaba que, en caso de ataque soviético, todo el armamento nuclear en manos de los Estados Unidos podría ser estallado en territorio chino, soviético, norcoreano y de los países satelitales. En 1958 los Estados Unidos usaron el misil nuclear llamado Atlas que estaba equipado con una bomba termonuclear W-38 que tenía suficiencia de 3,75 megatones (Fernández, 2015).

A partir de 1959, los revolucionarios cubanos se tomaron el poder de su país y empezaron a sostener diálogos con la Unión Soviética; por tal motivo, los Estados Unidos cortaron sus relaciones con el Gobierno cubano, y en 1962 se instalaron los misiles de mediano y corto alcance en la isla. La mayor parte de los misiles eran de tipo R14, que tenían la capacidad de transportar una ojiva nuclear de $3.000 \mathrm{~kg}$ a $3.000 \mathrm{~km}$; también se instaló el misil R-12, que tenía una bomba termonuclear con capacidad de 9,65 megatones (Fernández, 2015).

1 Ciudades del Imperio Japonés que fueron atacadas con bombas nucleares bajo la orden de Harry S. Truman, presidente de los Estados Unidos. 2 En EE. UU. a los planes para la utilización de armamento nuclear se les denominó SIOP, acrónimo de Special Integrated Operations Plan, plan especial de operaciones integradas.
Otro panorama fue el de la Guerra de Vietnam, en el cual se dio un enfrentamiento bélico de helicópteros y se pusieron en uso los fusiles de asalto M16 y AK-47. Este fue un conflicto entre guerrillas, que acabo con la teoría de que la batalla aérea solo se desarrollaría por medio de misiles; sin embargo, se volvió al cañón y la ametralladora como armas para derrotar al adversario (Fernández, 2015). Para ese escenario de combate, el Ejército de los Estados Unidos utilizó el cañón eléctrico M61 Vulcan, que, posterior a la guerra, fue reemplazado por el Gatling M197 de tres cañones como respuesta a la necesidad de aumentar la cadencia y poder de fuego, limitando el efecto de retroceso que estas armas generaban en aeronaves livianas y helicópteros.

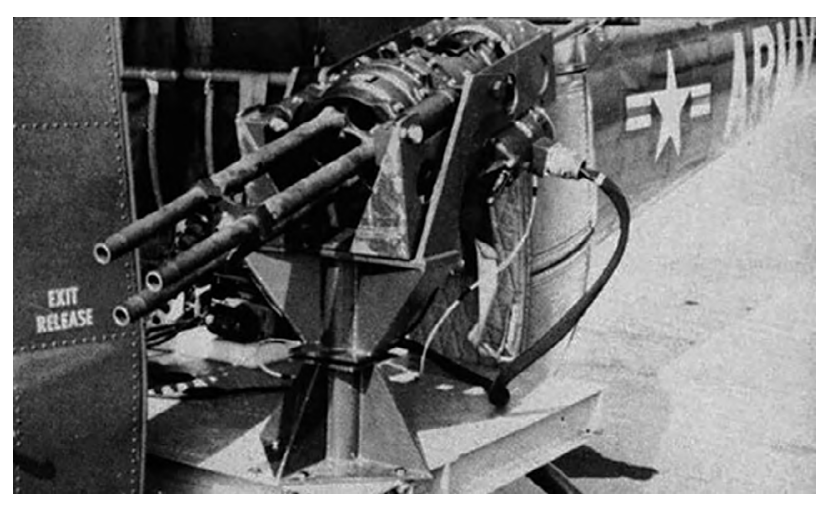

Figura 13. Cañón eléctrico Gatling M197 Fuente: Desarrollo y Defensa (2020).

Durante los años setenta entró en escena el cañón GAU-8 / A Avenger, como respuesta a la necesidad de un arma antitanque versátil, con capacidad de disparar municiones incendiarias de alta cadencia de fuego (3.900 disparos por minuto). Esta poderosa arma se montaba en una aeronave diseñada específicamente para tal fin, el Fairchild Republic A-10 Thunderbolt II, conocido como "Warthog" o "Hog".

Al igual que muchos países del mundo, Colombia también ha vivido la guerra en una geografía difícil e inhóspita por las variedades climáticas y vegetativas del país, en donde la Fuerza Aérea ha tenido que liderar operaciones determinantes para lograr el cese al fuego, el cual ha venido cambiando en cada una de las etapas transcurridas entre los siglos xx y XxI (Triana, 2016, pp. 378, 380, 385). 

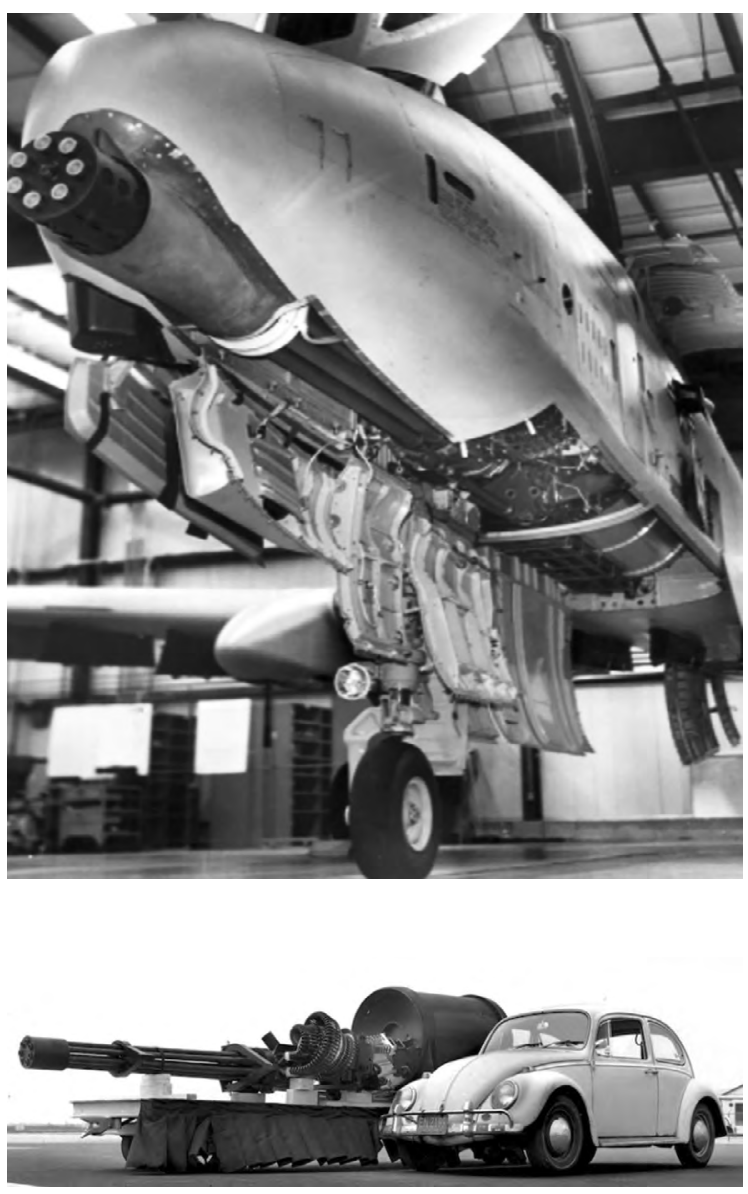

Figura 14. Cañón GAU-8 Avenger Fuente: Unidos (2015).

En 1939, las selvas colombianas del Putumayo y el Caquetá fueron el escenario de la barbarie cometida por la Casa Arana (explotadora de caucho) en el Perú. Las fronteras entre Colombia y el vecino país no estaban bien delimitadas, lo que condujo al enfrentamiento bélico. En aquella época Colombia contaba con una flotilla aérea de once aeronaves y el armamento para las Fuerzas Militares se componía de fusiles Máuser, algunos cañones y un limitado número de ametralladoras (ECURED, 2017). Con la debilidad militar que presentaba el país ante la amenaza peruana, se ordenó al cuerpo diplomático la adquisición del armamento en el exterior (Hernández, 2010).

Gracias a las relaciones diplomáticas, llegaron a Colombia 30 aeronaves Curtiss Hawk II F-11C, 22 Curtiss Falcon F-8F y 2 Commodore P2Y-1C de origen norteamericano; por parte de Alemania llegaron 17 Junker y 6 Dornier. Así, la Fuerza Aérea se fortaleció para enfrentar al Perú y a la vez proteger la soberanía nacional de cara al futuro (Hernández, 2010).

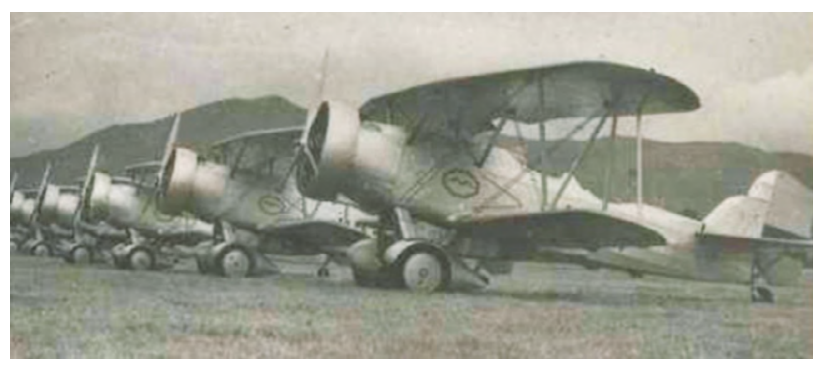

Figura 15. Curtiss Falcon F-8F, Fuerza Aérea Colombiana Fuente: Fuerza Aérea Colombiana (2020).

En 1953, arribaron a Colombia las primeras aeronaves de ala rotativa - helicópteros- $\mathrm{OH}-23$ Raven, y al año siguiente los primeros $\mathrm{OH}-13$ Sioux, acompañados de los Lockheed T-33 Silver Star. En 1956, ingresaron a Colombia las aeronaves Lockheed F-80 Shooting Star, seguidos de los bombarderos A-26C Invader, Canadair Sabre Mark IV, el Aero Commander L-26, el DHC2 Beaver, el C-45B Expediter y el C-54 Skymaster.

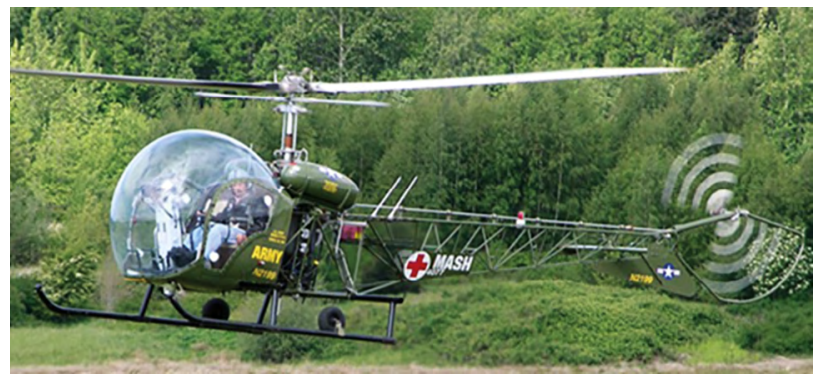

Figura 16. Helicóptero Bell Model 47 / H-13 Sioux Fuente: Heritage Fligth Museum (2020).

En 1963, llegaron los primeros UH-1B Iroquois, y poco después los primeros Cessna T-37 y T41 como preámbulo a la llagada de los Hughes OH6A Cayuse; durante 1969 llegó a Colombia la primera flota de UH$1 \mathrm{H}$ Huey (77 aeronaves); y 30 años después, Colombia adquirió los primeros KFIR, como punta de lanza de la defensa aérea del territorio colombiano. 


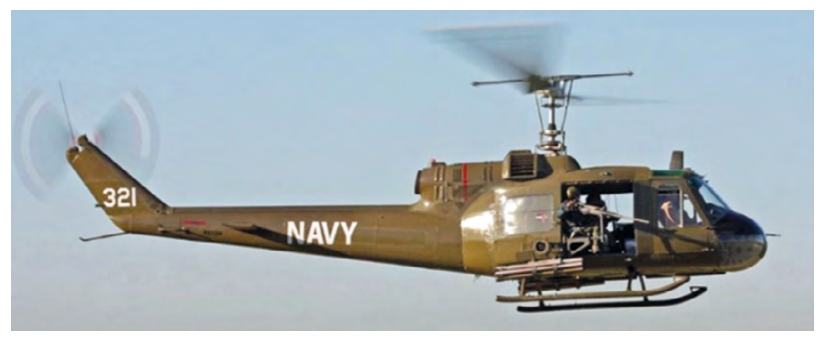

Figura 17. Helicóptero Bell Model Bell UH-1B

Fuente: War History Online (2020).

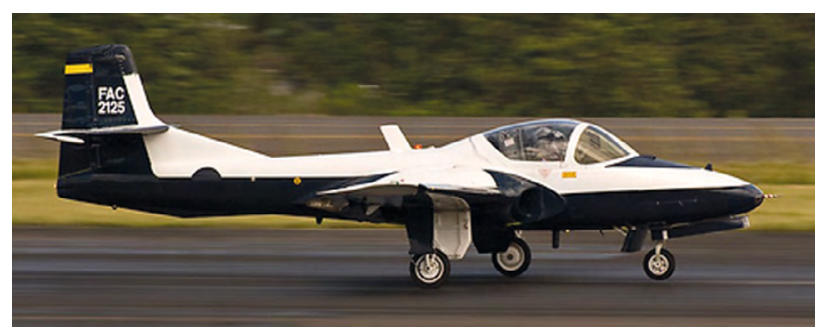

Figura 18. Cessna T-37B

Fuente: Badilla (2008).

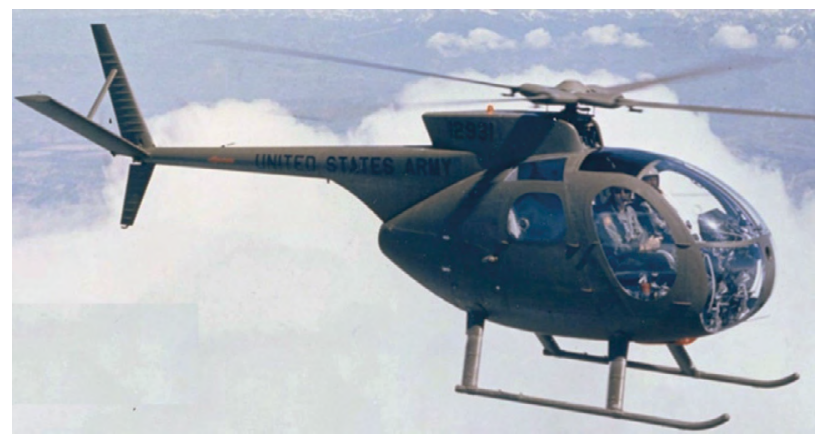

Figura 19. OH-6 CAYUSE / HUGHES 500

Fuente: Boeing Company (2020).

En los últimos 20 años el país ha enfrentado un fuerte conflicto interno contra grupos insurgentes, en donde el Ejército, la Armada Nacional, la Fuerza Aérea de Colombia y la Policía Nacional han tenido que realizar grandes operativos con el propósito de neutralizar a los grupos insurgentes; en 2015, los voceros del grupo revolucionario de las FARC indicaron, en La Habana, Cuba, que si hay algo a lo que le han temido, es a los bombardeos por parte de la fuerza pública.

Siguiendo los pasos de la historia, uno de los más importantes bombardeos ocurrió en 1990 durante la operación Colombia a Casa Verde en un campamento de las FARC en La Uribe (Meta). Para tal operativo, se utilizaron varias aeronaves cargadas con bombas de 250 libras. Entre las aeronaves que participaron hubo KFIR, AT-37 y AC-47, así como helicópteros artillados Bell 212, UH-60, UH-1H, Hughes, dos Bell ambulancia y un Hughes de comando y enlace (Escobar, 1995).

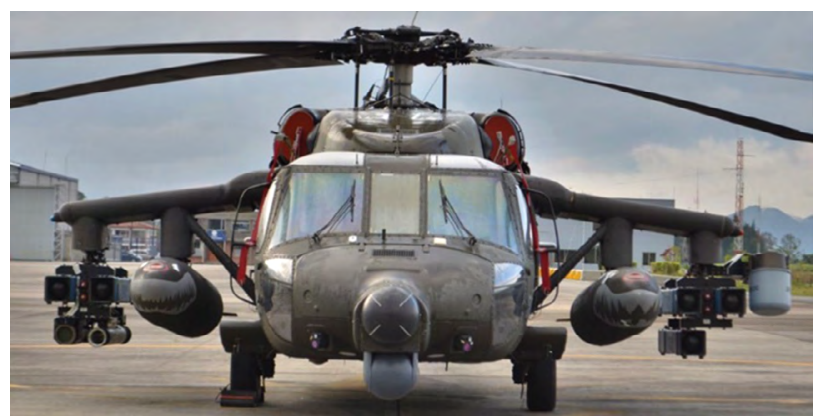

Figura 20. Helicóptero Sikorsky AH-60L Arpía IV

Fuente: Fuerza Aérea Colombiana (2020).

En septiembre del 2007, las Fuerzas Armadas desarrollaron en el departamento del Guaviare la operación Sol Naciente, en la que fallecieron Tomás Medina -alias el "Negro Acacio" - y 16 guerrilleros más (Revista Semana, 2010). En esa ocasión se utilizaron siete aviones Súper Tucano cargados con bombas de 500 libras y cinco helicópteros Black Hawk (El Tiempo, 2007). Al siguiente año, en un operativo denominado Operación Fénix, la Fuerza Aérea bombardeo parte del Putumayo cerca al territorio ecuatoriano, en donde murieron 21 guerrilleros y alias Raúl Reyes, segundo jefe de las FARC en aquella época

Otro operativo de gran importancia fue la Operación Sodoma, en la que murió uno de los hombres más buscados mundialmente, alias el "Mono Jojoy", durante un bombardeo de las Fuerzas Armadas en septiembre de 2010 en La Macarena, Meta. Durante el 2011, se dio otro golpe con la Operación Odiseo, que puso fin al máximo comandante de la guerrilla de las FARC, Guillermo León Sáenz Vargas, alias "Alfonso Cano" (El Espectador, 2011).

Estos son algunos de los operativos liderados por la Fuerza Aérea Colombiana, en los cuales se utilizaron aeronaves Súper Tucano cargados con bombas 
inteligentes. Gracias a la modernización de las Fuerzas Militares y la inteligencia técnica, se logró contribuir al inicio de los acercamientos de paz entre el Estado y el grupo revolucionario de las FARC.

Se puede analizar cómo, a partir del último tercio del siglo xx, empezó una nueva etapa de adelanto tecnológico, en donde el empleo de las comunicaciones, la informática, la tecnología láser, la electro-óptica, la robótica, los sensores y la tecnología espacial -que incluye el lanzamiento de misiles, los lanzamientos espaciales y los satélites - fueron imprescindibles para los sistemas de armas modernas e hicieron parte de la denominada revolución técnica militar, la cual representó un gran avance respecto al pasado (IEEE, 2014, pp. 18, 19).

De acuerdo con lo anterior, el ciberespacio y el espacio exterior tienen una dimensión estratégica asociada a los nuevos retos dentro del panorama de la seguridad global. Por ello, Colombia y la mayoría de los países necesitan de las nuevas tecnologías tanto en las actividades civiles como en las operaciones militares. Hoy en día los vehículos aéreos no tripulados, también llamados drones, se están convirtiendo en un elemento principal dentro de los ejércitos, por la rápida innovación tecnológica, relacionada con el control remoto y la robótica, que facilita el combate a distancia y las operaciones sin poner en riesgo vidas humanas (IEEE, 2014, p. 19).

\section{Conceptos de seguridad y defensa en Iberoamérica}

La realidad latinoamericana se puede observar mediante el análisis que demuestra que es bajo el índice de conflictividad entre los países; sin la existencia de armas nucleares, con un bajo presupuesto de inversión en gasto militar y visto desde una óptica estratégica no representa una amenaza para el orden mundial. Aunque es una región que tiene problemas de violencia de tipo regional que no repercuten - políticamente hablando- cuando las grandes potencias mundiales se reúnen para tratar temas de orden estratégico, Iberoamérica es vista como una zona de gran potencial para la producción de materia prima para las industrias de países desarrollados (Cobo, 2014). Además, cuando se analiza la situación geopolítica en América Latina, es fácil evidenciar los diversos actores y orientaciones políticas que tienen estos países, entre las que se encuentran corrientes capitalistas y socialistas, entre otras; las cuales, en muchos casos, basan su economía en el libre de mercado (Cobo, 2014).

Por otro lado, existe un fortalecimiento tecnológico de las aeronaves derivado del entendimiento de que el poder aéreo abarca espectros más amplios, como los del ambiente aéreo y espacial, que podría ser "ejercido a través del espacio por medio de misiles balísticos intercontinentales o por el uso del espacio con el empleo de satélites" (Ramos \& Oliveira, 2010), en donde las capacidades bélicas instaladas en las aeronaves son consideradas una proyección del poder nacional, ya sea como instrumento de acción política y militar, o "como factor de desarrollo económico y social, con el objetivo de conquistar y mantener los objetivos nacionales" (Ramos \& Oliveira, 2010).

De igual manera, las principales características para proyectar el poder bélico con el objetivo de conquistar y mantener el dominio del ambiente aéreo combativo se constituyen en la cantidad de aeronaves con capacidades de poder aéreo para enfrentar escenarios con alteraciones relacionadas con conflictos y escenarios donde posiblemente las aeronaves tradicionales con armamento convencional serán sustituidas por otras que se adapten a las nuevas demandas de seguridad, que se contrarrestan con armamento sofisticado y nuevas tecnologías militares (Ramos \& Oliveira, 2010).

Los "factores de planificación deben ser considerados para la preparación y el empleo del poder aéreo" (Ramos \& Oliveira, 2010), lo que implica proyectar los adelantos militares en materia de armas sofisticadas para mantener el dominio del aire encaminado a contrarrestar la amenaza actual del terrorismo en todas sus modalidades, las cuales pueden ser explicadas bajo los conceptos generacionales de las guerras (Ramos \& Oliveira, 2010). 


\section{Conceptos de seguridad para Colombia}

El mayor general Paredes (2011) considera que un país pacifista por naturaleza, como Colombia, debe construir su pensamiento estratégico sobre hipótesis de guerra, es decir, sobre peligros - por lejanos que aparezcan- de que intereses extraños puedan configurar amenazas que hagan necesaria la aplicación del poder militar. Para ese proceso existen numerosos factores condicionantes tales como la comparación del poder militar actual y potencial frente a posibles enemigos, la configuración geográfica de los presuntos teatros de guerra, la idiosincrasia de los pueblos, la capacidad y preparación de los mandos, entre otros cuantos en los espacios imponderables, es decir, en aquellos que no admiten cuantificación, sino apreciaciones teóricas o apreciaciones sobre el pasado bélico de pueblos y naciones.

La historia presentada en esta investigación nos muestra un pequeño panorama que indica que las naciones en cada una de las líneas de tiempo vividas han propendido por fortalecer, innovar y aplicar sus capacidades armamentísticas aéreas en pro de superar las amenazas existentes y prevenir factores de riesgo venideros (Paredes, 2011).

La industria militar aeronáutica en el ámbito mundial establece sus avances tecnológicos con base en las relaciones geopolíticas entre países, es decir, no se muestran aeronaves sobresalientes, como ocurre en la aviación comercial y sus reglas claras en el mercado de producción, sino que se definen las nuevas generaciones de aeronaves con armamento a partir de los objetivos estratégicos de las naciones que son protagonistas para ese momento (Ramos, 2010).

Battaleme (2009) explica las "causas y consecuencias de la creciente incorporación de nuevos sistemas de armas en el espacio regional" en Latinoamérica, a través del análisis del impacto y los efectos en las relaciones políticas y el desbalance regional que traen consigo los límites en la cooperación efectiva para afrontar preocupaciones de interés regional. En el caso particular de Colombia, obedece a las amenazas a la seguridad producto de fronteras porosas y no controladas por parte de los estados vecinos que, aunque cuentan con capacidades adquiridas, no son debidamente empleadas.

Cuando se incorpora nuevo armamento a las capacidades bélicas de un país, se genera, a su vez, una percepción de desbalance o carrera armamentística que trae consigo problemas y dilemas de inseguridad con tensiones de preservación y transformación de posibles escenarios de riesgo; los Estados no dejan de tener propensión a presentar dilemas de seguridad en relación con sus vecinos (Battaleme, 2009).

Los sistemas de armas incorporan cada vez más electrónica y despliegan más información sobre el campo de batalla, lo que permite que "sean empleadas más eficazmente con nuevas modalidades y en nuevas misiones" (Gutiérrez, 1995), jugando un papel fundamental en el diseño y producción, con un continuo crecimiento que afronta la complejidad de las guerras. A medida que las armas operan con mayor precisión y alcance, los sistemas computarizados incrementan el volumen de datos disponibles para la toma de decisiones, adquiriendo mayor importancia (Gutiérrez, 1995). Así mismo, cuando se aumenta el control de su operación, de la cadencia de fuego y la disminución del tamaño, se logra que las nuevas armas dispuestas en las aeronaves para la guerra moderna sean utilizadas para defender el espacio aéreo contra ataques estratégicos, o para combatir amenazas a la seguridad (Gutiérrez, 1995).

De igual manera, las capacidades tecnológicas en armas incorporadas a las aeronaves, elaboradas con nuevos materiales compuestos como fibras de vidrios, carbono y plásticos que compensan la resistencia, la fatiga y aumento de precisión, se convierten en tendencia al sustituir los utilizados tradicionalmente; estas mejoras brindan fiabilidad y avance en su implementación (Gutiérrez, 1995).

Los sistemas de armas incorporados en los aviones y helicópteros obedecen principalmente a los avances tecnológicos que producen mejoras en su utilización, y que son capaces de romper paradigmas y otorgar mejores prestaciones para su utilización, facilitando que los sistemas de armas le ayuden a los 
pilotos de combate a elegir la utilización del armamento acertado ante una situación complicada de combate (Gutiérrez, 1995).

\section{Consideraciones de seguridad para Colombia}

Colombia, pese a ser el cuarto país más grande de Latinoamérica y contar con alianzas para obtener armamento estadounidense, no tiene una flota de aeronaves de superioridad aérea moderna con armamento avanzado para enfrentar algún ataque extranjero y combatir actividades ilícitas como el narcotráfico y terrorismo (Pinzón, 2020).

De acuerdo con lo expresado por Pinzón (2020), "Los fenómenos de la guerra históricamente se han situado como un escenario de conquista, ambición territorial y expansión por parte de los Estados", lo cual conlleva a tensiones entre países que, aunque no promueven un enfrentamiento directo, tienen niveles de prevención ante cualquier hostilidad que se presente por un Estado oponente; estos países, además, reaccionan a través de la actualización de capacidades bélicas y del rearme (Pinzón, 2020).

Como muestra de lo anterior, en el 2009 se presentó en América Latina un importante "incremento en la adquisición de armas, en donde los Estados de Brasil, Colombia, Chile, México y Ecuador sobresalieron significativamente", bajo la justificación de afrontar los nuevos retos en materia de seguridad y competitividad disuasiva que le permitiera a cada país, con la compra de nuevas armas más sofisticadas, ser referentes en su poder y pensamiento político ante países vecinos (Pinzón, 2020). Así, cuando los países presentan mayor poderío militar y con fuerza generan posturas políticas, causan temor en otros; por su posición de actor político superior, traen consigo unas alertas de seguridad bajo el concepto de balance de poder (Pinzón, 2020).

La Fuerza Aérea Colombiana ha presentado un significativo avance con aviones equipados y modernizados que fortalecen los sistemas de inteligencia y planeamiento de operaciones militares que, de acuerdo con los cambios estratégicos y tecnológicos, han marcado el panorama mundial y son importantes para los ejércitos, puesto que con ello pueden realizar operaciones exitosas en contra del terrorismo o de los grupos insurgentes que pretendan colocar en riesgo la seguridad de cualquier país (Esquivel Triana, 2016).

En ese sentido, el equipo bélico es el fundamento material sobre el cual se edifican los operativos militares y las estrategias, de ahí la importancia de que la fuerza pública tenga armamento sofisticado para ofrecer diversas opciones de defensa y contrarrestar las posibles amenazas de intentos bélicos que se puedan presentar en el futuro y así mantener el orden y soberanía de una nación (Esquivel Triana, 2016).

En la actualidad, Colombia atraviesa por un periodo de postconflicto como consecuencia de la firma de paz con el principal grupo revolucionario del país, las FARC. Por tal motivo, el Estado colombiano se enfrenta a nuevos escenarios marcados por fenómenos de desmovilización, formación de nuevos grupos al margen de la ley, narcotráfico y minería ilegal, frente a los cuales la Fuerza Aérea Colombiana deberá demostrar todo su liderazgo con nuevas estrategias y operativos, haciendo uso de todo el avance tecnológico en el área del armamento aéreo para brindar dominio del espacio externo, ciberespacio y seguridad a la nación (Leal, 2017).

Por otro lado, Flavin (2003), en su artículo "Planificación de la terminación de conflictos y posconflicto exitoso", explica que la terminación de conflictos es el final de la lucha, pero no es el fin del conflicto. Indica, además, que el objetivo de las operaciones militares es establecer condiciones que obliguen a los beligerantes a poner fin a las hostilidades, mediante las aplicaciones de nuevas estrategias que permitan conservar la sana doctrina de aplicar las capacidades bélicas para combatir las amenazas que atenten contra la seguridad del Estado colombiano.

De igual manera, se puede concluir que el futuro de la carrera de fortalecimiento y aplicabilidad bélica aún está por recorrerse, y en este camino se debe visualizar bajo una constante aplicación de nuevas tecnologías de armamento en las aeronaves destinadas para la realización de operaciones militares (Flavin, 2003). 


\section{Recomendaciones para romper paradigmas}

Fomentar un liderazgo militar como forma de administración para lograr innovar en una creatividad que permita la constante aplicación de capacidades bélicas hacia capacidades creíbles (Pinzón, 2020).

Los líderes militares, en quienes recae la toma decisiones como una obligación de sus cargos, deben fortalecer su espíritu para abordar innovaciones que les permitan ser acertivos y visionarios en las capacidades bélicas que deben adquirir para Colombia (Pinzón, 2020).

La generación de cambios culturales debe estar apalancada con momentos sociales que brinden soporte argumentativo para tomar decisiones que permitan la consecución de nuevas capacidades bélicas (Pinzón, 2020).

Los Estados deben incrementar la actualización de las capacidades de armamento aéreo con nuevas tecnologías para las fuerzas armadas que permitan, dentro de la demostración de poder, ser referentes en la utilización aeronaves en el espacio aéreo con fines militares y promover ventajas estratégicas ente la resolución de crisis (Pinzón, 2020).

Colombia aún sigue presentando un aumento de la criminalidad derivada de los diferentes grupos al margen de la ley, que, vistos desde al ámbito militar, se deben contrarrestar con el uso de las armas. Desde los inicios, el poder aéreo y su utilización para la aplicación de la fuerza ha tenido muchas trasformaciones tecnológicas para beneficio de los Estados, al convertirse en fundamentales para la prevención y seguridad de los espacios aéreos (Pinzón, 2020).

\section{Conclusiones}

Las organizaciones militares a lo largo de la historia han enfocado sus esfuerzos en mejorar y sofisticar su armamento terrestre y aéreo en búsqueda de capacidades bélicas superiores, aplicables a diversos escenarios de conflicto.

Los avances bélicos del armamento aéreo dependen estrechamente del avance tecnológico de las mismas aeronaves. Aeronaves de mayores capacidades de vuelo, velocidades y autonomía promovieron el desarrollo de nuevos sistemas de armamento y el uso de estos.

Guerras de orden mundial se convirtieron en referentes en la validación de innovaciones incrementales como elementos efectivos y definitivos a la hora de superar y doblegar al enemigo.

Las innovaciones incrementales en sistemas de armamento han mejorado la efectividad de las operaciones militares aéreas; sin embargo, las condiciones de operaciones especiales en las que se desarrollan las mismas en el territorio nacional permiten que se puedan generar nuevos desarrollos "tropicalizados" a nuestras necesidades.

Colombia ha generado y validado desarrollos bélicos acorde a las duras exigencias de nuestro conflicto armado. Al igual que en las primeras guerras mundiales, los desarrollos tecnológicos del sector aeronáutico exigen que nuestras fuerzas armadas, apoyadas por la industria nacional, generen un nuevo y mejorado armamento aéreo que le permitan estar a la vanguardia en sistemas de seguridad y defensa.

Al considerar la evolución en las armas de fuego en comparación con los nuevos retos de seguridad para un país, surgen desafíos relacionados con la búsqueda de tecnologías adecuadas para ser empleadas en las aeronaves para combatir las amenazas persistentes o emergentes con las que cuenta un país.

Debe haber una constante actualización de las capacidades militares bajo el concepto de lineamentos diplomáticos que, en el contexto internacional, sea un referente de trasformación en material para la seguridad, y que satisfaga el desarrollo de un país y su confianza en el manejo de la política exterior (Pinzón, 2020).

La protección de una nación debe ser una obligación ante amenazas internas o externas en escenarios futuros; se debe buscar un equilibrio "mediante el poder con la adquisición de armamento militar" (Pinzón, 2020). 
La evolución tecnológica en armamento de tipo militar es cada vez mayor; el "equipamiento aéreo ha madurado significativamente convirtiéndose más eficaz, preciso y devastador", razón por la cual su implementación en la parte aérea garantizará el alcance de los objetivos propuestos por una nación por medio del ataque o la defensa (Pinzón, 2020).

La "modernización de tecnologías debe ser uno de los apuntes más importantes frente a la actualización de capacidades militares en un país"; debe permitir una ventaja militar en un conflicto de naturaleza bélica para el normal funcionamiento del ámbito económico, social y cultural de una nación (Pinzón, 2020).

Declaración de conflicto de interés: Los autores no manifiestan conflictos de interés institucionales ni personales.

\section{Referencias}

Ananthasayanam, M., Ibrahim, K., \& Muralidharan, M. R. (2005). Historical Evolution of the Military Fighter Airplanes Around the Twentieth Century", 43rd AIAA Aerosppace Science Meeting and Exhibit 10-13, 326-337. https:// doi.org/10.2514/6.2005-326

Bae Systems. (2020). Annual Report, Hadvilland DH2 con ametralladora Lewis.303 sobre nariz. Bae Systems. https:// www.baesystems.com/en/article/2020-full-year-results

Badilla, C. (2008, 29 de octubre). Cessna T-37B Tweety Bird. Airteamimages. https://www.airteamimages.com/cessnat-37-tweet_FAC2125_colombia---air-force_77883.html

Battaleme, J. (2009). Releyendo la compra de armas en la región y la reintroducción del dilema de seguridad. Miríada, 4. https://core.ac.uk/download/pdf/233941326.pdf

Boeing. (2020, 25 de agosto). B-17 Flying Fortress. https://www. boeing.com/history/products/b-17-flying-fortress.page

Boeing Company. (2020, 25 de agosto). OH-6 CAYUSE/HUGHES 500. Boeing. http://www.boeing.com/history/products/ oh-6-cayuse.page

Brew, A. (1993). Boulton Paul Aircaft since 1915. Putnam Aeronautical Books.

Brew, A. (2020). The P.29 Sidestrand. En A. Brew, Boulton Paul Aircraft Since 1915. Fonthill Media.

Cobo, I. F. (2014). Análisis crítico de la seguridad en Iberoamérica. Pre-Bie3, 3, 4. https://dialnet.unirioja.es/servlet/ articulo? codigo $=7641786$
Desarrollo y Defensa. (2020). Cañón Gatlin M197. Desarrollo y Defensa. https://desarrolloydefensa.blogspot.com/ 2019/05/canon-gatling-m197.html

ECURED. (2017, 3 de septiembre). Guerra colombo peruana. ECURED. https://www.ecured.cu/Guerra_colombo-pe ruana

El Espectador. (2011, 5 de noviembre). Éxito de Operación Odiseo, muerte de "Alfonso Cano". El Espectador. https: //www.elespectador.com/noticias/judicial/exito-deoperacion-odiseo-muerte-de-alfonso-cano/

El Tiempo. (2007, 5 de septiembre). La muerte de "siete vidas". El Tiempo. http://www.eltiempo.com/archivo/do cumento/CMS-3710030

Escobar, E. T. (1995, 21 de mayo). Operación casa verde. El Tiempo. http://www.eltiempo.com/archivo/documen to/MAM-330793\#

Esquivel Triana, R. (2016, enero-junio). La Fuerza Aérea Colombiana y el cese del conflicto armado (1998-2015). Revista Científica General José María Córdova, 14(17), 477-401. https://doi.org/10.21830/19006586.18

Fernández, O. C. (2015, 19 de agosto). El armamento soviético y norteamericano en el contexto de la Guerra Fría. http://intrahistoria.com/el-armamento-sovietico-y-nor teamericano-en-el-contexto-de-la-guerra-fria/\#_Toc426 479996

Flavin, W. (2003). Planning for Conflict Termination and Pos-Conflict Success. Parameters, autumn, 96-112.

Fuerza Aérea Colombiana. (2020, 25 de agosto). Fuerza Aérea Colombiana. https://www.fac.mil.co/fuerza-a\%C3\%A 9rea-colombiana-lanza-arp\%C3\%ADa-iv

Gutiérrez, L. (1995). Evolución de la tecnología military “su impacto" en España. Cuadernos de Estrategia, 75, 83-114.

Heritage Flight Museum. (2020, 25 de agosto). H13 Sioux. Heritage Flight Museum. https://heritageflight.org. Obtenido de https://heritageflight.org/aircraft/h13-sioux/

Hernández, D. (2010). La aviación colombiana en la guerra contra el Perú. Pago, 84-89. http://www.au.af.mil/au/afri/ aspj/apjinternational/apj-s/2010/2010-2/2010_2_09_ hernandez.pdf

Hernández Sampieri, R., Fernández Collado, C., \& Baptista Lucio, P. (2010). Metodología de la investigación. McGraw-Hill.

Hiveminer. (2020). Hiveminer. www.hiveminer.com

IEEE. (2014). Cuaderno de estrategias 169 desarme y control del armamento del siglo xxI: limitaciones al comercio y a la transferencia de tecnología. Instituto Español de Estudios Estratégico. http://www.ieee.es/Galerias/fiche ro/cuadernos/CE_169.pdf 
Leal, B. F. (2017, 3 de julio). Expectativas en el posacuerdo. El Espectador. http://colombia2020.elespectador.com/ politica/expectativas-en-el-posacuerdo

Loftin, L. K. (1987). Quest for Performance: the Evolution of Modern Aircraft. NASA Scientific and Technical Information Branch. https://doi.org/10.2307/3105034

Militarylmages. (2007, 5 de enero). Militarylmages. https:// www.militaryimages.net/media/boulton-paul-p-29-si destrand-crew-1931-raf-101sqn.14974/

Pallarés, B. M. (1984). La guerra electrónica y la electrónica en la guerra. Boletín de Información, (171), 3.

Paredes, L. (2011). Seguridad y estrategia: los desafíos del futuro. Fuerzas Armadas de Colombia.

Pinzón, J. (2020). Colombia frente a la actualización de capacidades militares suramericanas: el caso del sistema de defensa antiaéreo [Tesis de pregrado, Pontificia Universidad Javeriana]. https://repository.javeriana.edu.co/ bitstream/handle/10554/53913/Tesis\%20Juli\%C3\% A1n\%20David\%20Pinz\%C3\%B3n\%20G\%C3\%B3mez. pdf? sequence $=1$

Ramos, F., \& Oliveira, P. (2010). Cambios en el perfil de la guerra y reflexiones para la preparación y el empleo del Poder Aéreo Brasileño, Revista profesional-Fuerza Aérea de EUA. https://www.airuniversity.af.edu/Portals/10/ JOTA/Journals/Volume\%201\%20Issue\%201/03-Fernan dez_s.pdf

Revista Semana. (2010, 23 de junio). Tres años de golpes a las FARC. Revista Semana. https://www.semana.com/ nacion/articulo/tres-anos-golpes-farc/122355-3

Richard, J. (2009, 14 de diciembre). History of War. http://www. historyofwar.org/Pictures/pictures_boulton_paul_ overstrand.html

San Diego Air \& Space Museum. (2020, 13 de julio). San Diego Air \& Space Museum. https://sandiegoairandspace.org/

SilverHawkAuthor. (2020). Ametralladora en el plano superior. http://silverhawkauthor.com/canadian-warplanes5-ontario-ottawa-canadian-war-museum_867.html
Strong F. J., \& Jaramillo J. M. (2014). La Fuerza Aérea Colombiana y la Acción Integral, doctrina y política de Estado [Trabajo de grado].

The Museum of Fligth. (2020, 25 de agosto). A Short History of the $B$-17. https://blog.museumofflight.org/a-short-his tory-of-the-b17

Triana, R. E. (2016). La Fuerza Aérea Colombiana y el cese del conflicto armado (1998-2015). Revista Científica General José María Córdova, 14(17), 377-401: http://www.scielo. org.co/pdf/recig/v14n17/v14n17a14.pdf

Unidos, M. N. (2015, 7 de abril). National Museum. https:// www.nationalmuseum.af.mil/Visit/Museum-Exhibits/ Fact-Sheets/Display/Article/197393/martin-b-10/

Wageningen. (2008). Vanwageningen.net. https://www.van wageningen.net/picture/2914-20080528_64_rnlaf_ hawker_hunter_f4_n_144_military_aviation_museum_ nl/category/46-2008_military_aviation_museum_at_ kamp_zeist_the_netherlands

War History Online. (2020, 7 de abril). "Huey" For Sale, With Amazing History. War History Online. https://www.war historyonline.com. https://www.warhistoryonline.com/ military-vehicle-news/huey.html

WonderfulEngineering. (2020). Dardos Aéreros. https://won derfulengineering.com/before-bombs-were-inventedpilots-dropped-these-steel-arrows-on-the-enemy/

Wright State University. (2020, 25 de agosto). The Dayton-Wright Airplane Company. https://corescholar.libraries.wri ght.edu/special_ms152_photographs/120/

Zimmerman, D. J. (2019). Top Gun: 50 Years of Naval Air Superiority. Quarto Publishing Group USA Inc. https:// books.google.com.co/books?id=hjeSDwAAQBAJ\&pg= $\mathrm{PA} 111 \& \mathrm{dq}=$ synchronous + machine+gun+mounted+ on+a+aircraft+fokker\&hl=es-419\&sa=X\&ved=2ahUKEwi 1sa3H2c_qAhXmRd8KHcl3CK0Q6AEwAHoECAEQAg\# $\mathrm{v}=$ onepage $\& \mathrm{q}=$ synchronous $\% 20$ machine $\% 20$ gun $\% 20$ mounted\%20on\%20a\%20aircraft\%20f 